\title{
Spirochaete pallida in den Plasmazellen bei der progressiven Paralyse.
}

\author{
Von \\ Dr. Henry Marcus, \\ Dozent der Psychiatrie am Karolinischen Institut zu Stockholm. \\ Mit 1 Textfigur und 1 Tafel. \\ (Eingegangen am 14. Mai 1914.)
}

Noguchi ist es zuerst im Februar 1913 gelungen, typische Spirochäten in der Hirnrinde von Paralytikern nachzuweisen. Lange war es ja schon von den meisten Forschern erkannt, daß die Paralyse ausschließlich bei Syphilis vorkommt. Das Verhalten zwischen der syphilitischen Infektion und der sog. Parasyphilis blieb doch immer schwierig befriedigend $\mathrm{zu}$ erklären. Die neueren serologischen Untersuchungen, die Wassermannsche Reaktion usw. machten es aber mehr und mehr wahrscheinlich, daß es bei der Paralyse sich um eine syphilitische Spirochätose handle.

Nachdem Leva diti gezeigt hatte, daß es mit der Silberimprägnierung leicht gelinge, die Spirochäten in syphilitischen Läsionen nachzuweisen, haben sowohl Levaditi wie mehrere Forscher zahlreiche, aber erfolglose Versuche gemacht, die Spirochäten im Gehirn der Paralytiker zu entdecken. Sie hatten den Versuch aufgegeben, und beinahe hatte Noguchi dies auch getan, als er in einer Untersuchungsreihe von 70 Präparaten endlich in dem 69. eine typische Spirochaete pallida beobachtete. Er machte nun weitere Untersuchungen von 200 Gehirnen und fand Spirochäten, aber nur in $25 \%$ der Fälle. Seine Befunde wurden nun bald von mehreren Forschern bestätigt und die Präparate, von denen auch ich von Noguchi bekommen habe, zeigen alle typische Spirochäten und typische paralytische Veränderungen der Hirnrinde. Sie sind schön gefärbt mit der von Nog u chi angegebenen, modifizierten Levaditischen Argentummethode.

Einstweilen, ob nun auch hierdurch sicher konstatiert worden war, daß die Spirochäten sich bei der Paralyse im Gehirn oft finden lassen, blieben doch die häufigen negativen Befunde zum Teil unerklärlich. Levaditi, Marie und Bankowski konnten in ihren gemeinsamen Untersuchungen die Spirochäten mit der Silbermethode zuerst nur in 10\% demonstrieren. Es kam so der Verdacht, daß die Spirochäten

Z. f. d. g. Neur. u. Psych. O. XXVI. 
nicht immer bei der Paralyse zu finden seien, sondern daß deren Vorkommen eine zufällige Kombination mit einer direkten Syphilis des Gehirns bedeute. Dieser Verdacht scheint nun beseitigt zu sein durch die neueren Untersuchungen von Levaditi, Marie und Bankowski, die durch Benutzung einer besonderen Technik und durch systematische Untersuchungen jeder Hirnwindung dazu gekommen sind, daß sie erklären können, daß es gelinge, in der größten Mehrzahl, wenn nicht sogar in allen Fällen von Paralyse, die Spirochäten nachzuweisen.

Sie untersuchen die Hirnrinde so bald wie möglich nach dem Exitus, ohne Härtung, und teils direkt mit dem Ultramikroskop, teils mittels Chinatuschfärbung oder anderen Färbungen, die in der Arbeit näher beschrieben sind. Sie haben mit diesen Methoden Spirochäten in reichlicher Zahl in den meisten untersuchten Fällen angetroffen. Sie fanden sich hauptsächlich in den hinteren Teilen der Frontalwindungen.

Es scheint, daß es vorzugsweise die schnell verlaufenden Fälle sind, bei denen man hoffen kann, die Spirochäten zu finden. Die Dauer der Paralyse war von einem bis vierzehn Monate. Und vor allem ist es bei solchen Kranken, die im Anfall gestorben sind, wo man die Spirochäten findet. Diese Behauptung macht auch Noguchi.

Die Menge der Spirochäten variiert höchst bedeutend in den verschiedenen Fällen. In einigen waren sie so reichlich, wie in der Primärsklerose, in anderen wieder so sparsam, daß man lange suchen mußte, um eine zu finden. Besonders bemerkenswert war, daß die Spirochäten in mehr oder weniger circumscripten Herden zusammen lagen.

Wie Noguchi schon beschrieben, fanden auch Levaditi u. a., daß sie in der Rinde liegen, und daß die weiße Substanz gewöhnlich frei ist.

Was die genauere Verteilung in der Rinde betrifft, so fand Nog u chi sie ziemlich regelmäßig zwischen den Nervenzellen und den Neurogliafasern verteilt. Zuweilen beobachtete er, daß die Spirochäten sich der Nervenzelle anlegten. In der Pia mater fand er sie nicht, und, was bemerkenswert ist, nur ganz selten in der Nähe von Blutgefäßen, und fast niemals in den Gefäßwandungen.

Levaditi u. a. a. haben jedoch in ein paar von den in der erwähnten Arbeit beschriebenen Fällen gesehen, daß die, Spirochäten in intimer Relation zu den Gefäßwandungen waren, und haben sie auch in der infiltrierten Pia nachweisen können.

In einem Präparate, das ich von Prof. Noguchi zugesandt bekommen habe, liegen auch einige Spirochäten in sehr naher Beziehung zu den Gefäßen, in deren adventitiellen Räumen.

Wie bekannt, ist ja im pathologisch-anatomischen Bilde der progressiven Paralyse das Verhalten der adventitiellen Lymphscheiden von der größten diagnostischen Bedeutung. Wie besonders durch $\mathrm{Alz}$ heimers und Nissls Untersuchungen gezeigt wurde, sind sie bei fast 
allen Fällen von Paralyse durch große, eigentümliche Zellen, hauptsächlich sog. Plasmazellen, ausgefüllt. Diese bilden einen dichten Mantel um das Gefäß herum. Besonders bei den stürmisch verlaufenden Fällen ist dieser Mantel enorm.

Es ist ja sicher, daß diese massenhafte Zelleninfiltration eine große Bedeutung haben muß für die paralytische Erkrankung, und daß dieselbe eine wichtige Stütze ist für diejenige Ansicht, daß die syphilitische Noxe zuerst die Gefäße und erst später die Nervensubstanz angreift. Es schien mir deshalb am wahrscheinlichsten, daß gerade diese Zelleninfiltration der hauptsächlichste Fundort der Spirochäten sein werde, und es war mir deshalb ganz sonderbar, daß Nog u chi sie gerade in der Nähe der Gefäße so oft vermißt hatte. Wie erwähnt, haben ja aber Levaditi und seine Mitarbeiter sie später dort öfters gefunden. Ich habe nun auch, wie andere Untersucher, mehrere Gehirne von Paralytikern vergebens untersucht und bin überzeugt, daß der negative Befund oft auf Ungewohnheit bei der Technik beruht. Denn die Methode erfordert viel Mühe und Geduld. Einstweilen ist es auch mir nun gelungen, in einem Falle von typischer progressiver Paralyse deutliche, wenn auch nur sparsame Spirochäten demonstrieren zu können. Warum ich aber meinen Befund hier erwähne, ist deshalb, weil ich die Spirochäten in typischen Plasmazellen eingeschlossen gefunden habe. Ich beschreibe hier kurz den Fall.

E. K., 46 Jahre. Gest. 1907. Kaufmann.

Vor mehreren Jahren hatte Pat. eine syphilitische. Infektion, die während der nächsten Zeit ziemlich gründlich behandelt wurde. Seit 1895 hat er an Tabes gelitten und im Jahre 1904 brach die Psychose ziemlich plötzlich hervor. 1905 hatte er eine ziemlich gute Remission, aber 1906 trat die Krankheit von neuem hervor, eingeleitet durch paralytische Krampfanfälle. Er wurde nach diesen zuerst verwirrt, unruhig und auch gewalttätig. Nachher entwickelte sich eine zunehmende Schwäche der Gedankentätigkeit und des Gedächtnisses. Es trat allmählich eine schwere gemütliche Reizbarkeit hinzu.

Die Sprache wurde langsam und stolpernd, die Pupillen ohne Reaktion und die Patellarreflexe waren verschwunden. Der Patient erhielt kräftige $\mathrm{Hg}$-Behandlung, aber der Zustand verschlimmerte sich mehr und mehr; Halluzinationen und hypochondrische Wahnvorstellungen traten hinzu. Die Gedankentätigkeit wurde mehr und mehr inkohärent. Das Gedächtnis nahm mehr und mehr ab. Auch trat zuweilen eine leichte Euphorie ein.

Im I907 häuften sich die paralytischen Anfälle. Nach sehr zahlreichen Krämpfen am 14. IV. 1907 trat um 3 Uhr nachmittags der Exitus ein.

Sektion 15. IV., 9 Uhr vormittags.

Die Dura ist bedeutend verdickt, zeigt eine Pachymeningitis interna. Die Pia ist milchig verdickt, besonders in den frontalen Teilen, und dort an mehreren Stellen mit der Gehirnoberfläche fest vereint und läBt sich dort nicht ohne Substanzverlust des Gehirns fortziehen. Bedeutende Atrophie der Gyri des Frontalhirns.

Das Rückenmark zeigt typische tabische Veränderungen.

Mikrosko pische Untersuchung.

Schnitte aus der Frontalrinde zeigen eine verdickte Pia, die stark mit Plasma- 
zellen und Rundzellen infiltriert ist. Man sieht oft, wie diese infiltrierte Pia Wurzeln tief in die Gehirnoberfläche hineinsendet. Die marginale Glia ist sehr verdichtet und enthält zahlreiche Astrocyten. Die intracerebrale Glia ist so stark entwickelt, daß der Bau der Rinde kaum zu erkennen ist. Stäbchenzellen finden sich einzeln im Bilde. Die Nervenzellen zeigen verschiedene bei der Paralyse als charakteristisch angesehene Veränderungen, und ein bedeutender Untergang von Nervenzellen hat stattgefunden.

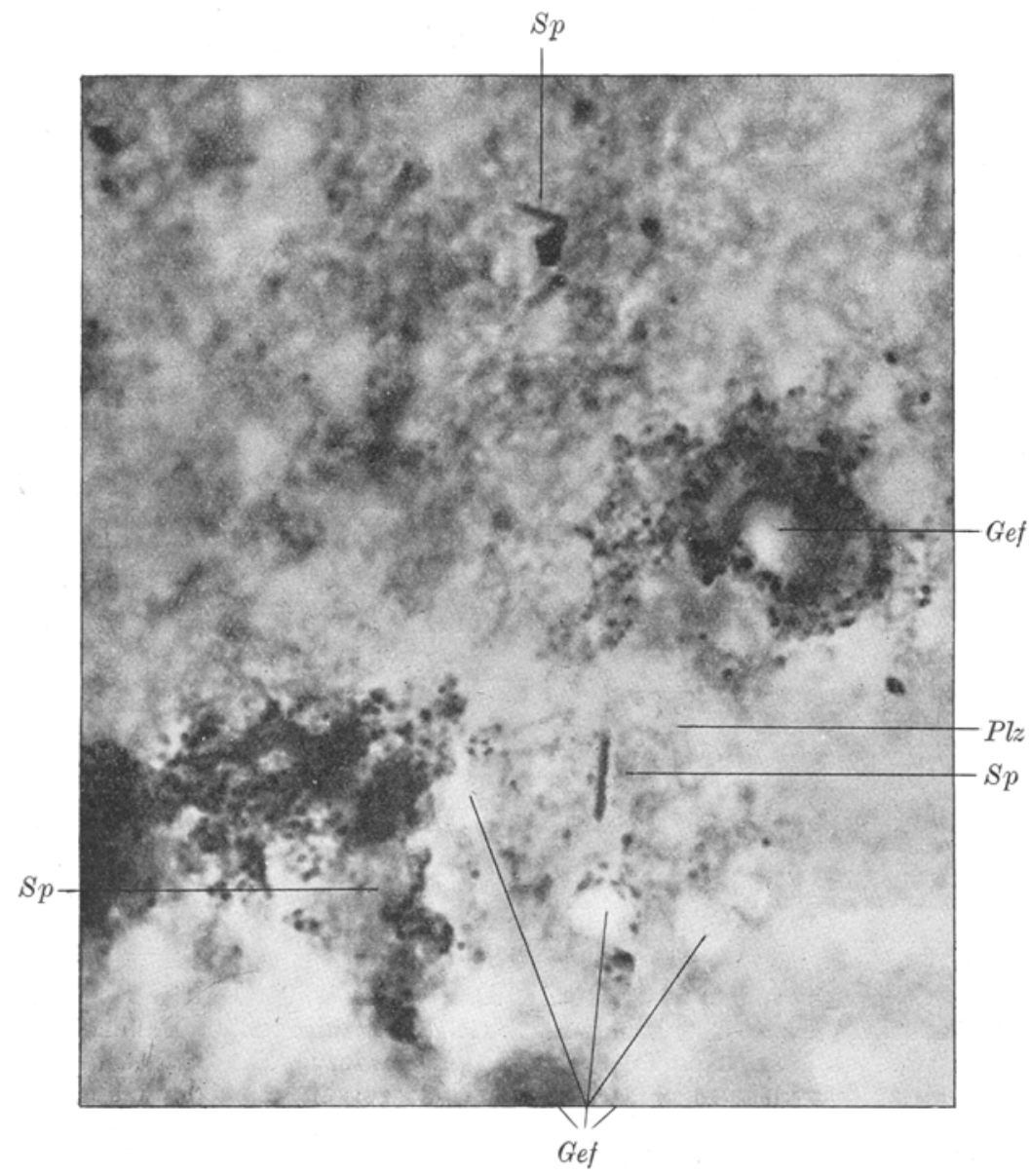

Fig. 1. $S p=$ Spirochaete pallida. Gef $=$ GefäBlumen. $P l z=$ Plasmazelle.

Eine reichliche Neubildung von Gefäßen findet sich überall in den Schnitten. Keinen sklerotischen Prozeß findet man an den kleinen Gefäßen und nirgends circumscripte Zellenherde im Gewebe. Dagegen zeigen alle Gefäße eine ganz massenhafte Ausfüllung der adventitiellen Räume, durch hauptsächlich größere und kleinere Plasmazellen, die überall dicke Mäntel bilden um das Gefäß. Verschiedene Plasmazellen erblickt man auch hier und da in den äußeren Teilen der grauen Substanz frei in der Nähe von kleinen Gefäßen zwischen Glia- oder Ganglienzellen. 
In den Präparaten, die nach der von Nog uchi modifizierten Levaditischen Silbermethode behandelt sind, habe ich nun innerhalb einiger Plasmazellen deutliche Spirochäten nachweisen können.

Diese Plasmazellen liegen teils dicht an der Gefäßwand, teils in sehr kurzer Entfernung von derselben. Die Lokalisation der Spirochäten geht hervor aus den beistehenden Bildern, von denen Fig. 1 eine Mikrophotographie ist von einem Präparate aus der Frontalrinde des Falles E. K. Tafel I, Fig. 1 ist eine Zeichnung von derselben Stelle, und Tafel I, Fig. 2 stellt ein Bild aus einem anderen Präparate desselben Falles dar. Die Mikrophotographie ist an der hiesigen histologischen Institution von Prof. E. Holmgren aufgenommen, die anderen Bilder von Frl. S. Forsgren an derselben Institution gezeichnet.

An der Mikrophotographie Fig. 1 sieht man einige Spirochäten, von denen die in der Mitte sich in Tafel $\mathbf{Y}$, Fig. 1 wiederfindet. Da das meiste am Noguchi-Präparat ja stark entfärbt ist, kann man die Konturen der Plasmazellen an der Photographie nicht sehr deutlich sehen. Die stärker gefärbten Stellen sind teils Körner im Plasma oder Kerne verschiedener Plasmazellen, teils Blutkörperchen im Gefäß. Wenn man aber die 3 an der Photographie markierten Spirochäten etwas näher beobachtet, sieht man, wie die Spirochäten unten links und die, die sich im oberen Teile des Bildes befinden, in Verbindung mit Zellen sind, die dicht am Gefäßlumen liegen. Die Spirochäte in der Mitte des Bildes liegt dagegen etwas vom Gefäß entfernt, und die Zellgrenze tritt an der Photographie etwas undeutlich hervor. In Tafel I, Fig. 1, wo diese Spirochäte besonders gezeichnet ist, sieht man aber besser, wie das Gefäß von zahlreichen Plasmazellen umgeben ist, und wie eine von diesen eine Spirochaeten pallida, die deutlich gefärbt ist und die typischen Windungen zeigt, enthält. Der größte Teil der Spirochäte befindet sich im Plasma der Plasmazelle, ein kleinerer Teil ragt in einen rundlichen, hellen Raum hinein, der vielleicht ein Querschnitt des gewundenen naheliegenden Gefäßes ist oder auch ein erweiterter lymphoider Raum.

Tafel I, Fig. 2 zeigt auch ein mit Blutkörperchen gefülltes Gefäß von Plasmazellen umgeben. In einer großen Plasmazelle, die etwas vom Gefäß entfernt ist, sieht man eine Spirochäte, die nach verschiedenen Einstellungen des Mikroskops gezeichnet ist, da sie sich nicht in einer Ebene befindet. Sie liegt zum größten Teil dicht über dem Kern der Plasmazelle, zum Teil zur Seite des Kerns im Plasma und streckt sich auch aus der Zelle hinaus.

In einer Plasmazelle sah ich eine Spirochäte im Zellenplasma ganz eingeschlossen, in einiger Entfernung vom Kern liegend; in einer anderen Plasmazelle schmiegte sich die Spirochäte genau an die Kerngrenze an.

Die Spirochäten kamen nur einzeln vor und sehr sparsam. Ich habe 
sie nur in einem begrenzten Bezirk in der Frontalrinde in deren 2. und 3. Schicht angetroffen, teils in Plasmazellen, teils frei im Gewebe. In den Ganglienzellen und Gliazellen sowie in der Pia habe ich sie nicht gesehen.

Bei Durchmusterung des Präparates von Noguchi habe ich auch dort eine Stelle gesehen, wo eine Spirochäte sich mit einem Teile in einer Plasmazelle dicht an der Gefäßwand befindet, während der übrige Teil frei im adventitiellen Gewebe liegt.

Wenn es sich nun in ferneren Untersuchungen zeigen sollte, daß dies kein einzelner Befund ist, sondern daß die Spirochäten regelmäßig in den Plasmazellen vorkommen bei der Paralyse, scheint es mir, daß vielleicht diesen Zellen als Träger der Spirochäten bei der Paralyse eine wichtige Aufgabe zukommt. Einerseits können sie als Reaktionszellen auftreten zur Aufnahme und ev. Zerstörung des aus den Gefäßen hervordrängenden Spirochätenzugs, andrerseits können einzelne Plasmazellen, denen diese Zerstörung nicht gelingt, in das Gewebe hinein weiter wandern mit ihrer Spirochätenlast. Diese kann nun an die Ganglienzellen abgegeben werden, wo Noguchi sie ja nachgewiesen hat, dort ihre deletäre Wirkung ausüben und die paralytische Erkrankung schließlich hervorrufen.

Der Nachweis, den Levaditi u. a. geführt haben, daß Spirochäten in der meningitischen Infiltration vorkommen, zeigt ja außerdem, daß wahrscheinlich auch von dieser äußeren Seite her ein zusammenwirkender Eingriff auf das Gehirn ausgeübt werden kann, um schließlich eine progressive Paralyse hervorzurufen.

\section{Literaturverzeichnis.}

Noguchi, Münch. med. Wochenschr. Nr. 14. April 1913.

- Paralysie générale et Syphilis. La Presse médicale Nr. 76, 81. Sept. et Oct. 1913.

Levaditi, Marie et Bakowski, Annales de l'Institut Pasteur. Juillet 1913. Marinesco et Minea, ref. in Zeitschr. f. d. ges. Neurol. u. Psych. Ref. Bd. 9, H. 4 . 